those with and without Sentinel Node Biopsy (SNB) in the first procedure.

Methodology Retrospective cohort study involving 27 patients diagnosed with low-risk EC (ESMO-ESGO-ESTRO criteria) with surgical restaging due to upstage in the final histological result at the Hospital Universitario Donostia from April 2013 to September 2018. Surgical and oncological results were compared between patients who underwent hysterectomy and double adnexectomy without any additional procedure (SNB-) $\mathrm{n}=17$ and those who also had a pelvic and aortic SNB (SNB +) $n=10$. The main outcome evaluated in the study was intraoperative complications. Secondary outcomes were mean operative time, length of hospital stay, number of nodes obtained, Progression-Free Survival (PFS) and Overall Survival (OS).

Results The median duration of restaging surgery was 240 minutes (Q25 - Q75: 180 - 300) in the SNB(-) group, and 300 (Q25 - Q75: 247.5 - 330) minutes in the $\mathrm{SNB}(+)$ group, this difference being statistically significant (one-side $\mathrm{t}$-student test, $\mathrm{p}=0.0295$ ). With regard to intraoperative complications, there were $17.65 \%$ vs $40 \%$ respectively, all of them vascular, this difference being not significant. There were no statistical differences in length of hospital stay and number of pelvic nodes obtained. PFS and OS in both groups were the same.

Conclusion Women with EC who require lymph node restaging due to upstage and have previously undergone sentinel lymph node biopsy have greater surgical difficulty with longer duration of the procedure. The risk of complications is increased.We advise against performing a second re-staging surgery in patients sentinel node biopsy.

Disclosures No disclosures.

\section{HAVE WE IMPROVED UTERINE SEROUS CARCINOMAS MANAGEMENT THE LAST TWO DECADES? A SINGLE CENTER EXPERIENCE}

${ }^{1}$ Michail Liontos, ${ }^{2}$ Anna Svarna, ${ }^{2}$ Roubini Zakopoulou, ${ }^{2}$ Flora Zagouri, ${ }^{3}$ Charalampos Theofanakis, ${ }^{3}$ Nikolaos Thomakos, ${ }^{2}$ Dimitrios Haidopoulos, ${ }^{4}$ Alexandros Rodolakis, ${ }^{5}$ Maria Sotiropoulou, ${ }^{6}$ Meletios-Athanasios Dimopoulos, ${ }^{6}$ Efi Skafida, ${ }^{6}$ Maria Kaparelou, ${ }^{6}$ Konstantinos Koutsoukos. 'General Hospital of Athens 'Alexandras'; ${ }^{2}$ National and Kapodistrian Unviersity of Athens; Department of Clinical Therapeutics; ${ }^{3}$ University of Athens; Alexandra Hospital; 1st Department of Obstetrics and Gynecology; ${ }^{4}$ National and Kapodistrian University of Athens; 1st Department of Obstetrics and Gynecology; ${ }^{5}$ Department of Pathology, Alexandra Hospital; ${ }^{6}$ Department of Clinical Therapeutics, National and Kapodistrian University of Athens, Alexandra Hospital

\subsection{6/ijgc-2020-ESG0.76}

Introduction/Background Uterine Serous Carcinomas (USC) account for ten percent of endometrial cancers. Management of the disease is challenging due to its aggressiveness. Despite numerous clinical trials and advances in the molecular pathology of the disease it remains the most lethal uterine carcinoma. We undertook this study to determine changes in the management of the disease and the survival of our patients the last two decades.

Methodology Medical records of women with USC treated at Alexandra Hospital from 2000 to 2018 were retrospectively identified. Clinicopathological data, treatment and survival data were analyzed. Kaplan-Meier Survival curves were generated using Strata SE 15.1; survival differences were estimated using the long-rank test.
Results 121 patients with USC and complete clinical data were identified. Median age was 66.9 years. Pure serous carcinomas were diagnosed in 66 patients, while the remaining had mixed histologies. At diagnosis, 33 patients $(28.0 \%)$ had stage Ia disease, but also 28 patients had stage IV disease (23.7\%). 115 patients were treated with surgery including lymphadenectomy in 63 cases $(55.8 \%)$ and omentectomy in 83 cases $(73.5 \%)$. 64 patients $(52.9 \%)$ were treated from 1999 to 2009. There was no statistical difference in the stage distribution, omission of lymphadenectomy or omentectomy and type of adjuvant treatment among patients treated prior or after 2010. Also, for patients with stage I-III disease, eligible for adjuvant treatment, both disease free survival and overall survival (OS) per stage did not differ significantly between the two decades. Finally, in patients with de novo stage IV disease there was no improvement in either first line Progression Free Survival or OS between the two decades (mPFS 1st vs 2nd decade: 8.6 vs 5.9 months 95\% CI [1.5-15.7].[3.4-8.5] and mOS 18.4 vs 11.6 months 95\% CI [1.7-34.9].[0.0-25.1].

Conclusion Despite recent clinical trials, surgical and medical management of USCs had not changed significantly the last two decades in everyday clinical practice. This is reflected in the survival of the patients.

Disclosures Authors have no conflict of interest.

\section{ENDOMETRIAL CANCER: THE ROLE OF PROGNOSTIC FACTORS AND THEIR IMPACT ON RECURRENCE PATTERN}

${ }^{1}$ Giulia Parpinel, ${ }^{2}$ Luca Fuso, ${ }^{1}$ Maria Elena Laudani, ${ }^{1}$ Annalisa Carapezzi, ${ }^{2}$ Enrico Badellino, ${ }^{2}$ Nicoletta Petronio, ${ }^{3}$ Marisa Ribotta, ${ }^{1}$ Martina Barboni, ${ }^{2}$ Annamaria Ferrero, ${ }^{1}$ Paolo Zola. ${ }^{1}$ Sant'anna Hospital; Department of Surgical Sciences; ${ }^{2}$ Mauriziano Hospital; Department of Surgical Sciences; ${ }^{3}$ Aou Città Della Salute e Della Scienza; Department of Pathological Anatomy

\subsection{6/ijgc-2020-ESG0.77}

Introduction/Background The rate of recurrence of endometrial cancer is $11-19 \%$. It is related to different prognostic factors which define specific risk classes in order to decide for an adjuvant treatment. The objective of this study is to evaluate how prognostic factors influence the probability and pattern of recurrence.

Methodology This multicentric observational retrospective study was conducted on 552 patients treated for endometrial cancer between February 2011-2019. The considered parameters were: age, BMI (Body Mass Index), surgery, stage, LVSI (Lymphovascular Space Involvement), myometrial infiltration, histological grade, lymph node involvement, adjuvant therapy, relapse. DFS (Disease Free Survival) and OS (Overall Survival) were stratified by the presence or absence of prognostic factors.

Results The rate of recurrence was $14,7 \%$. Median time to recurrence was 15 months and 5 -years OS was $84 \%$. We observed an increase from 6 to $30 \%$ in the distance metastasis rate associated with positive LVSI. Monovariate analysis showed a correlation between DFS and advanced stage (O.R. II 6.7; III-IV 9.2 $\mathrm{p}=0,0001$ ), positive lymph nodes (O.R. 3.7; $\mathrm{p}=0,04)$, myometrial infiltration (O.R. $3.9 \mathrm{p}=0,0001)$ and LVSI (O.R. $3.5 \mathrm{p}=0,005)$. Similar results were observed considering $O S$ and grading $(p=0,002)$. We conducted a Cox 